Z. klin. Chem. u. klin. Biochem.

9. Jg., S. $497-502$, November 1971

\title{
Enzymatische Glucosebestimmung in hämolysierten Blutproben ohne Störung durch Fructose
}

\author{
Von F. DA FONSECA-WOLLFEIM
}

\begin{abstract}
Aus dem Institut für Klinische Chemie und Kliniscbe Biochemie (Direktor: Prof. Dr. H.-J. Dulce) am Klinikum Steglitz der Freien Universität Berlin
\end{abstract}

(Eingegangen am 16. Juli 1971)

Glucose kann im Hāmolysat nach Ausschaltung störender Erythrocytenenzyme zuverlāssig mit der Hexokinasemethode über die NiADPHFluoreszenz bestimmt werden. Inaktivierung der Erythrocyten-Phosphoglucose-Isomerase, und damit Eliminierung der bisher problematischen Fructoseinterferenz, wird auf einfache Weise durch Hāmolyse mit 0,1v Essigsäure erreicht. Die beschriebene Methode zeichnet sich durch geringen Zeitaufwand und ökonomische Reagenziennutzung aus und eignet sich auch besonders für Routineuntersuchungen.

\section{The enzymic determination of glucose in baemolysed blood samples without interference by fructose}

Glucose can be reliably determined in exythrocyte haemolysates by the hexokinase method and the measurement of the NADPH fluorescence after the elimination of interfering erythrocyte enzyme activities. The erythrocyte phosphoglucose isomerase, which hitherto has caused the problematic interference by fructose, is inactivated by simply carrying out the haemolysis in $0.1 \mathrm{x}$ acetic acid. The described method is characterised by its short time of operation and the economic use of reagents and it is especially suitable for routine investigations.

Die enzymatische Glucosebestimmung direkt im Hämolysat kann für die Praxis wesentliche Vorteile bieten. Der Fortfall der Enteiweißung bei der Blutzuckerbestimmung bedeutet Zeitersparnị und Eliminierung einer Fehlerquelle aus dem Arbeitsgang.

Durch den störenden Einfluß von Erythrocytenenzymen ist allerdings die Hexokinasemethode bei Anwendung auf Hāmolỵsat ohne zusātzliche Maßnahmen zu unspezifisch (1). Das bei der Reaktionsfolge

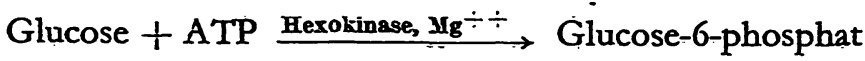
+ ADP

$$
\begin{aligned}
\text { Glucose-6-phosphat } & + \text { NADP }^{+} \frac{\text { Glucose- }}{\begin{array}{l}
\text { 6-Phospat- } \\
\text { Dehsdrogenase }
\end{array}} \rightarrow \text { Gluco- } \\
& \text { nat-6-phosphat }+\mathrm{NADPH}+\mathrm{H}^{+}
\end{aligned}
$$

gebildete Gluconat-6-phosphat wird durch Erythrocyten-6-Phosphogluconat-Dehydrogenase unter Bildung von NADPH weitèr umgesetzt, was sich in einem fortlaufenden Extinktionsanstieg nach Abschluß der eigentlichen Bestimmungsreaktion äußert (2). Durch Zusatz des Enzyminhibitors Maleinimid konnte diese Störung beseitigt werden $(1,3)$. HAECKEL machte aber auf die verbleibende, im klinischen Bereich nicht ungefāhrliche Miterfassung von Fructose aufmerksam (1). Wir beschreiben im folgenden Versuche zur Entwicklung einer Methode, die auch bei Anwesenheit von Fructose im Hāmolysat korrekte Ergebnisse liefert.

\section{Methodik}

Reagenzien

1. 0,3м Triāthanolaminpuffer $\mathrm{pH} 7,5$; $4 \mathrm{mar} \mathrm{MgSO}_{4}$

2. $12 \mathrm{mMNADP}$

3. $16 \mathrm{mM}$ ATP

4. $1 \mathrm{mg} / \mathrm{ml}$ Hexokinase; $1 \mathrm{mg} / \mathrm{ml}$ Glucose-6-phosphat-Dehydrogenase

Bestandreile der Böhringer-Testkombination TGAA oder TGAB

5. $20 \mathrm{mg} / \mathrm{ml}$ Maleinimid (EGA-Chemie). Bei $4^{\circ}$ einige Wochen haltbar.

6. $0,1 \times$ Essigsāure

7. Glucose-Stammlösung 4,00 g/1:3,80 g Glucose (wasserfrei) ad 1000,0 mit dest. Wasser lösen. Der um 5\% gegenüber dem Nennwert verminderte Gehalt kompensiert die durchschnittliche Fluoreszenzminderung bei Blutproben (s. u.).

8. Glucose-Standard-Gebrauchslösung: Stammlösung 1:21 mit $0,1 \mathrm{~N}$ Essigsäure verdünnen.

9. Reaktionsgemisch:

$1,5 \mathrm{ml}$ Lösung 1

$0,5 \mathrm{ml}$ Lösung 2

$0,5 \mathrm{ml}$ Lösung 3

$1,0 \mathrm{ml}$ Lösung 5

werden gemischt. 1 Woche bei $4^{\circ}$ stabil.

\section{Messung der NADPH-Fluoreszenz}

Für Fluoreszenzmessungen wurde das Turner-Fluorimeter Modell 110 mit Standardmeßtür benutzt. Blende 3x; Hg-Lampe mit Primärfilter 7/60 (360 nm Narrow Pass); Sekundärfilter 4 (465 nm Sharp Cut). Das Gerät wird mit Reagenzienleerwert in der Küvette auf Null abgeglichen. 


\section{Untersuchungsmaterial}

Wir verwenden zur Blutzuckerbestimmung Oxalat-Fluoridstabilisiertes Venenblut, das in entsprechend präparierten Spezialgefäßen angelicfert wird. Bei sofort nach Entnahme erfolgender Hämolyse (s. u.) kann auch mit Kapillarblut gearbeitet werden.

Durchführung der Bestimmung

\section{Hämolyse}

$50 \mu \mathrm{l}$ Blut werden mit $1,00 \mathrm{ml} 0,1 \mathrm{~N}$ Essigsäure hämolysiert. Unter diesen Bedingungen bleibt die Glucosekonzentration 24 Stunden bei Raumtemperatur unverändert.

\section{Bestintmungsansätze}

Auf den Boden des Inkubationsgefäßes (Einweg-Plastikröhrchen $17 \times 90 \mathrm{~mm}$ mit Verschlußkappe') werden mit Marburg-Pipetten dosiert:

\begin{tabular}{|c|c|c|c|}
\hline & Probe & Standard & Leerwert \\
\hline \multirow{4}{*}{$\begin{array}{l}\text { Reaktionsgemisch, } \mu \mathrm{l} \\
\text { Hämolysat, } \mu 1 \\
\text { Glucosestandard, } \mu \mathrm{l} \\
0,1 \text { Essigsäure, } \mu \mathrm{l} \\
\text { Für Vermischung sorgen! } \\
\text { Enzymsuspension, } \mu \mathrm{l}\end{array}$} & 100 & 100 & 100 \\
\hline & $\underline{20}$ & $\overline{20}$ & $\overline{-}$ \\
\hline & - & - & 20 \\
\hline & 5 & - 5 & 5 \\
\hline
\end{tabular}

Mischen. Frühestens nach 3, spätestens nach 15 Min. Inkubation bei Raumtemperatur $10 \mathrm{ml}$ dest. Wasser $\left(25^{\circ}\right)$ zusetzen, Röhrchen verschließen und durch Umkippen mischen. (Das zur Verdünnung zugesetzte Wasser wird bei konstanter Temperatur gehalten, um bei der Fluoreszenzmessung definierte Temperaturbedingungen in der Küvette zu gewährleisten. Ohne diese Maßnahme könnte die Temperaturabhängigkeit der Fluoreszenz (vgl. (4)) zur Fehlerquelle werden.)

Leerwertansatz in die Küvette gießen und Nullabgleich des Fluorimeters durchführen.

Küvette durch Absaugen mit der Wasserstrahlpumpe entleeren. Fluoreszenz von Probe und Standard messen (Küvette jeweils mit Meßlösung vorspülen).

Bexechnung

$$
\frac{\text { Fluoreszenz: }}{\text { Fluorese }} \times 4,00=\mathrm{g} \text { Glucose } / \mathrm{l} \text {. }
$$

Bei nicht hämoglobinhaltigen Proben Ergebnis mit 0,95 multiplizieren.

\section{Ergebnisse}

\section{Wahl des Meßverfahrens}

Meßgröße für den Glucosegehalt der Probe ist bei der Hexokinasemethode das gebildete NADPH. Für seine Erfassung stehen zwei Möglichkeiten zur Auswahl: Extinktionsmessung oder Fluorimetrie.

Die Voraussetzungen für den „optischen Test“ bei $366 \mathrm{~nm}$ sind bei Hämolysat ungünstig. Die Extinktion des Probenleerwertes liegt bei einer Probenendverdünnung von $1: 200$ und einer Schichtdicke von $1 \mathrm{~cm}$ etwa bei 1,0. Unter den gleichen Bedingungen erhält man z. B. bei einer Glucosekonzentration von $0,5 \mathrm{~g} / 1 \mathrm{im}$ „optischen Test" nur eine Extinktionsänderung von 0,046 . Es werden daher für präzises Arbeiten hohe Anforderungen an die Meßgenauigkeit und Konstanz des Photometers gestellt.

Eine der Probenleerwertextinktion bei der Photometrie entsprechende hohe Eigenfluoreszenz findet sich bei

1) Hersteller: Ernst Bechthold und Sohn D 6124 Beerfelden/ Odenwald. der Fluorimetrie von Hämolysat nicht. ProbenleerwertAnsätze (Hämolysat in Puffer) liefern nur sehr geringe, meist unterhalb der Nachweisgrenze liegende Fluoreszenzintensitäten (siehe Tab. 1). Bei der von BERNT und LACHENICHT angegebenen Methode zur mechanisierten Glucosebestimmung im Hämolysat (5) wurde dies bereits vorausgesetzt und direkte Proportionalität zwischen Fluoreszenz (abzüglich, des Reagenzienleerwertes) und Glucosegehalt angenommen. Wir haben an mehreren Hundert Blutproben aus allen klinischen Bereichen diese Frage überprüft und dabei nie eine Eigenfluoreszenz feststellen können, die einer Glucosekonzentration von mehr als $0,05 \mathrm{~g} / 1$ entsprach. Im Mittel lag die Leerwertfluoreszenz bei 0,15 Skalenteilen und zeigte damit eine scheinbare Glucosekonzentration von etwa $0,01 \mathrm{~g} / \mathrm{l}$ an. Wegen dieser günstigen meßtechnischen Voraussetzungen ist die Fluorimetrie für die Erfassung des NADPH im Hämolysat zu bevorzugen.

$\mathrm{Zu}$ berücksichtigen ist hierbei allerdings die hämoglobinbedingte Minderung der NADPH-Fluoreszenz: Gibt man zu einer NADPH-Lösung in Puffer Hämolysat, so nimmt die Fluoreszenz merklich $a b$, und $z w a r$ bei einer Hämolysatkonzentration, wie sie in unserem Testansatz verwirklicht ist, um etwa 5\%. Bei der in Tabelle 1 niedergelegten Versuchsreihe betrug die

Tab. 1

Einfluß von Hämolysat auf die gemessene NADPH-Fluoreszenz $\mathrm{Zu} 5 \mathrm{ml}$ einer NADPH-Lösung in Triäthanolaminpuffer $\mathrm{pH} 7,5$ wurden in der Fluorimeterküvette $20 \mu \mathrm{l}$ Hämolysat (Blutprobe $1: 42$ in $0,1 \mathrm{~N}$ Essigsäure) gegeben. Die Fluoreszenz wurde unter Standardmeßbedingungen vor (Spalte 2) und nach (Spalte 3) Hämolysatzusatz gemessen. Die Fluoreszenzabnahme unter Berücksichtigung der ġeringen bzw. unterhalb der Meßempfindlichkeit liegenden Probenleerwerte (Hämolysat in Puffer, Nullabgleich des Fluorimeters mit Puffer, Spalte 4) zeigt Spalte 5

\begin{tabular}{ccccc}
$\begin{array}{c}\text { Nr. der } \\
\text { Blutprobe }\end{array}$ & $\begin{array}{c}2 \\
\text { NADPH } \\
\text { ohne } \\
\text { Hämolysat }\end{array}$ & $\begin{array}{c}\text { NADPH } \\
\text { mit } \\
\text { Hämolysat }\end{array}$ & $\begin{array}{c}\text { Hämolysat } \\
\text { in Puffer }\end{array}$ & $\begin{array}{c}\text { Fluores- } \\
\text { zenzab- } \\
\text { nahme }\end{array}$ \\
\hline
\end{tabular}
- Skalenteile -

\begin{tabular}{rrrrr}
1 & 83,5 & 79,0 & 0,0 & 4,5 \\
2 & 84,5 & 81,0 & 0,5 & 4,0 \\
3 & 84,5 & 81,0 & 0,0 & 3,5 \\
4 & 84,5 & 80,0 & 0,0 & 4,5 \\
5 & 84,5 & 80,5 & 0,0 & 4,0 \\
6 & 83,5 & 79,0 & 0,5 & 5,0 \\
7 & 84,0 & 79,5 & 0,0 & 4,5 \\
8 & 84,0 & 80,0 & 0,0 & 4,0 \\
9 & 84,0 & 80,0 & 1,0 & 5,0 \\
10 & 83,5 & 79,5 & 0,5 & 4,5 \\
11 & 83,0 & 79,0 & 0,0 & 4,0 \\
12 & 83,5 & 79,5 & 0,0 & 4,0 \\
13 & 83,0 & 79,0 & 0,0 & 4,0 \\
14 & 83,5 & 79,5 & 0,0 & 4,0 \\
15 & 83,0 & 79,0 & 0,0 & 4,0 \\
16 & 83,5 & 79,5 & 0,0 & 4,0 \\
17 & 83,5 & 79,0 & 0,5 & 5,0 \\
18 & 84,0 & 79,5 & 0,0 & 4,5 \\
19 & 83,5 & 79,0 & 0,0 & 4,0 \\
20 & 84,0 & 80,0 & 0,0 & 4,0 \\
\hline
\end{tabular}

durchschnittliche Fluoreszenzabnahme 5,08\%, die Standardabweichung $\mathrm{s}=0,48 \%$. Wegen der nur geringen Schwankungsbreite nehmen wir bei hämoglobinhaltigen Proben eine generelle Korrektur vor (unter Verzicht auf das individuelle Zubereiten interner Glucosestandards bei jeder einzelnen Probe), indem wir die Glu- 
cosestandardlösung um $5 \%$ gegenüber dem Nennwert verdünnen. Rechnerischer Ausgleich ist ebenfalls möglich.

Um dic Fluoreszenzminderung möglichst gering zu halten, messen wir bei hoher Probenendverdünnung $(1: 10500)$. Die Methode ist daher nur mit einem ausreichend empfindlichen Fluorimeter anwendbar.

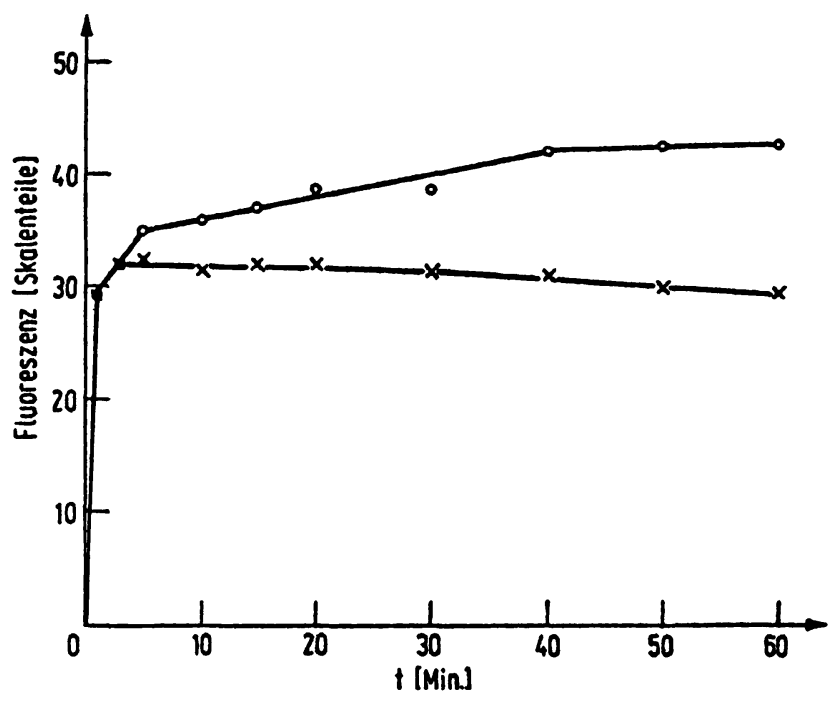

Abb. 1

Einfluß von Maleinimid auf die Meßwertkonstanz bei der fluorimetrischen Glucosebestimmung im Hămolysat. O-O-O ohne Maleinimid; $x-x-\times$ mit Maleinimid. Aus einem 10 fachen Inkubationsansatz wurden zu den eingezelchneten Zeitpunkten $100 \mu$ wurde die Fluoreszenz unter StandardmeBbedingungen bestimmt

Hemmung der Erythrocyten-6-Phosphogluconat-Dehydrogenase mit Maleinimid

Durch Oxydation von Gluconat-6-phosphat an Erythrocyten-6-Phosphogluconat-Dehydrogenase (EC 1.1.1.44) kommt die Fluoreszenzentwicklung nach vollstāndigem Umsatz des Glucose-6-phosphats nicht zum Stillstand. Abbildung 1 zeigt das Ausmaß dieser Störreaktion unter den Bedingungen unseres Testansatzes. Zusatz von Maleinimid oder $\mathbf{N}$-Äthylmaleinimid $(1,3)$ führt zur Inaktivierung der 6-Phosphogluconat-Dehydrogenase, und man erhält so einen über etwa $30 \mathrm{Min}$. konstanten Meßwert. Bei längerem Stehenlassen des Ansatzes nimmt die Fluoreszenz wieder leicht ab (siehe Abb. 1).

Um Fehler durch unvollständige Inaktivierung der 6-Phosphogluconat-Dehydrogenase zu vermeiden, wurde die Hemmwirkung von Maleinimid auf das Enzym unter variierten Bedingungen untersucht. Bei diesen Versuchen wurde Hãmolysat mit Maleinimid vorinkubiert. Anschließend wurde ein Aliquot in einem fluorimetrischen Aktivitätstest mit zúgesetztem Gluconat-6-phosphat auf noch vorhandene 6-Phosphogluconat-Dehydrogenaseaktivität getestet.

Die $\mathrm{pH}$-Abhängigkeit der Hemmstoffwirkung zeigt Tabelle 2. Vollständige Hemmung des Enzyms wurde bei schwach alkalischem $\mathrm{pH}$ erreicht. Dagegen blieb bei niedrigerem $\mathrm{pH}$ ein Teil der Enzymaktivität erhalten. Dies entspricht den Beobachtungen bei anderen Enzymen (6).
Tab. 2

pH-Abhăngigkeit der Hemmwirkung von Maleinimid auf Erythrocyten-Phosphogluconat-Dehydrogenase. Hämolysat in $0,01 \mathrm{M}$ Phosphatpulfer wurde an der Glaselektrode auf das angegebene pH eingestellt. Dann Zusatz von Maleinimid (Endkonzentration $2 \mathrm{mg} / \mathrm{ml}$ ) und 5 Min. Inkubation bei $25^{\circ} .50 \mu$ l des Ansatzes wurden entnonimen und mit $1,0 \mathrm{ml}$ Reaktionsgemisch für den 6-Phosphogluconat-Dehydrogenase-Test $(0,6 \mathrm{~mm}$ Oluconat-6-phosphat; $2 \mathrm{mM}$ NADP; $3,3 \mathrm{~mm}$ MeSO,; $0,25 \mathrm{M}$ Triathanolaminpuffer pH 7,5) 5 Min. inkubiert. Dann Zusatz von $5 \mathrm{ml} \mathrm{H}_{2} \mathrm{O}$ und Fluoreszenzmessung unter Standardme $\$$ bedingungen. Leerwerte ohne Oluconat-6-phosphat. Die Enzymaktivităt wird ausgedrückt als Fluoreszenzzunahme in Skalenteilen pro 5 Min. In der Spalte rechts ist die errechnete Restaktivităt, bezogen auf einen Ansatz ohne Maleinimid, angegeben

\begin{tabular}{ccc}
\hline pH & $\begin{array}{c}\text { Fluoreszenz- } \\
\text { zunahme/5 Min. } \\
\text { (Skalenteile) }\end{array}$ & \% Aktivität \\
\hline 5,5 & 29,0 & 92 \\
6,5 & 15,5 & 49 \\
7,5 & 0,0 & 0 \\
$\begin{array}{c}8,5 \\
\text { Ohne } \\
\text { Maleinimid, } \\
\text { pH 7,5 }\end{array}$ & 0,0 & 0 \\
\hline
\end{tabular}

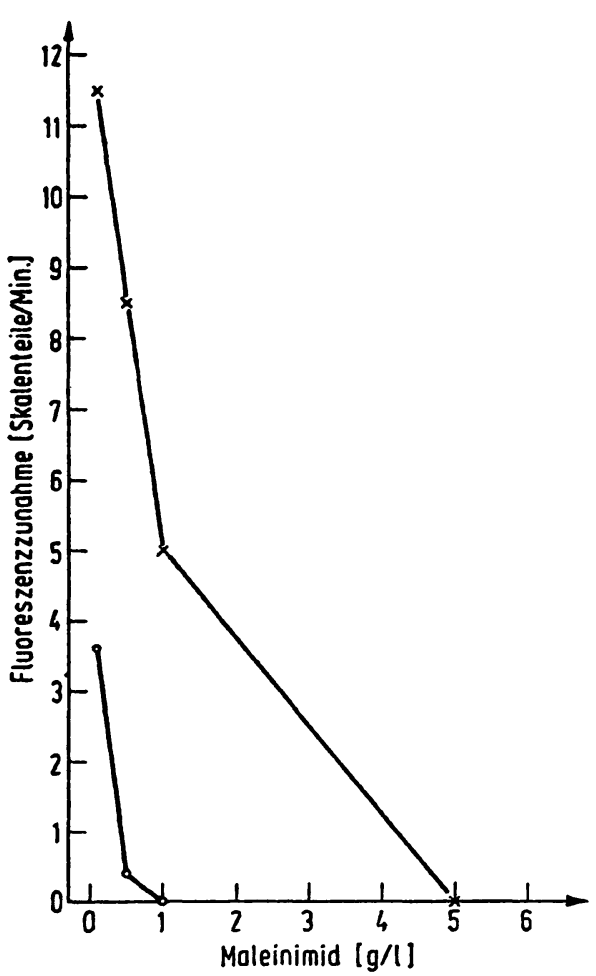

Abb. 2

Abhānsigkeit der 6-Phosphogluconat-Dehydrogenase-Inaktivierung von der Maleinimidkonzentration

Die nach 1- bzw. 5-minütigem Einwirken von Maleinimid bei pH 7,5 verbliebene 6-Phosphogluconat-Dehydrogenase-Aktivität ist in $A B-$ verbliebene 6-Phosphogluconat-Dehydrogenase-Aktivität ist in Abaufgetragen. $\times-X-X 1$ Minute; $0-0-05$ Minuten Vorin6-Phosphogluconat-Dehydrogenase-Test wie bei Tabelle 2 beschrieben

Weiterhin wurden die Parameter Hemmstoffkonzentration und Dauer der Einwirkung variiert. Wie $\mathrm{Ab}$ bildung 2 zeigt, muB für eine komplette Inaktivierung innerhalb einer Minute eine relativ hohe Maleinimidkonzentration gewählt werden. Bei längerer Einwirkung vor Start der Testreaktion kommt man mit einer geringeren Inhibitorkonzentration aus.

Inaktivierung der Erythrocyten-Phosphoglucose-Isomerase

Nach Beobachtungen von HAECKEL (1) werden Glucosebestimmungen im Hämolysat durch Fructose ge- 
stört. Hefe-Hexokinase phosphoryliert auch Fructose, und Fructose-6-phosphat kann durch ErythrocytenPhosphoglucose-Isomerase (EC 5.3.1.9) in Glucose6-phosphat umgelagert werden. Bei Hämolyse mit $\mathrm{H}_{2} \mathrm{O}$ ohne weitere Zusätze fällt die Miterfassung von Fructose in unserem Testsystem erheblich ins Gewicht siehe (Abb. 3).

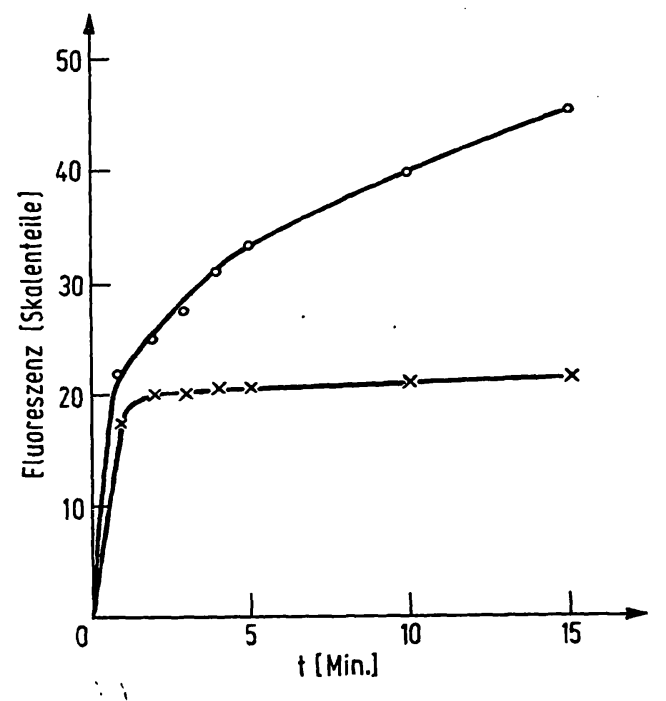

Abb. 3

Fluorimetrische Glucosebestimmung in fructosehaltigem Hämolysat. Fructosekonzentration $5 \mathrm{~g} / \mathrm{l}$. Aus 10 fachen Bestimmungsansatzen (siehe unter "Methodik") wurden zu den angegebenen Zeitpunkten $100 \mu \mathrm{l}$ entnommen, mit $10 \mathrm{ml} \mathrm{H}_{2} \mathrm{O}$ verdünnt und fluorimetriert. - -0 mit dest. Wasser bereitetes Hämolysat; $x-x-x$ mit 0,1 N Essigsäure bereitetes Hämolysát

$\mathrm{Da}$ Fructose in der Klinik häufig verabfolgt wird, mußte eine Möglichkeit zur Ausschaltung der Erythrocyten-Phosphoglucose-Isomerase gesuicht werden. Zunächst prüften wir die Brauchbarkeit der in der Literatur (7) beschriebenen kompetitiven Hemmstoffe des Enzyms. Bei diesen orientierenden Hemmversuchen diente als $\mathrm{Maß}$ für die Phosphoglucose-Isomeraseamtivität im Hämolysat die Fluoreszenzzunahme fructosehaltiger Testansätze zwischen der 5. und 15. Minute (vgl. Abb. 3). Bezogen auf die Aktivität des Kontrollansatzes ohne Inhibitor ergaben sich die folgenden Beziehungen:

$23 \mathrm{~mm}$ ATP

$11,5 \mathrm{~mm}$ Phosphoenolpyruvat

2,5 mM Desoxyglucose

6.5 ms Gluconat-6-phosphat

\section{$\%$ Aktivität}

43

44

8

Mit $20 \mathrm{~mm}$ Gluconat-6-phosphat war keine Phosphoglucose-Isomerase-Aktivität mehr meßbar. Unter diesen Bedingungen war der Glucose-Umsatz nicht beeinträchtigt und ein Einfluß auf die mit wäßr. Glucoselösungen erhaltenen Eichkurven nicht festzustellen. Gluconat-6-phosphat kann dem Testansatz aber erst dann zugesetzt werden, wenn die Erythrocyten-6Phosphogluconat-Dehydrogenase komplett inaktiviert ist (s. o.). Für eine Routinemethode erschien uns daher die Anwendung von Gluconat-6-phosphat als zu riskant.
Tab. 3

Inaktivierung von Erythrocyten-Phosphoglucose-Isomerase in $0,1 \mathrm{M}$ Acetatpuffer von unterschiedlichem pH. Hamolysat wurde 5 Min bei den angegebenen pH-Werten inkubiert ${ }_{\text {Phosphoglucose-Isomerase-Test (12) }}$

\begin{tabular}{cc}
$\mathrm{pH}_{4,2}$ & $\Delta \mathrm{E}_{\mathbf{g}, \mathrm{g} / \mathrm{Min} .}$ \\
3,9 & 0,020 \\
3,6 & 0,001 \\
& 0,042 \\
\hline in dest. Wasser & .1
\end{tabular}

Eine bessere Möglichkeit zur Ausschaltung des störenden Enzyms fanden wir bei der Untersuchung der Säurelabilität der Erythrocyten-Phosphoglucose-Isomerase. Hält man Hämolysat kurze Zeit bei einem $\mathrm{pH}$-Wert von unter 4, so sind beim anschließend durchgeführten optischen Phosphoglucose-Isomerase-Test nur noch weniger als $2 \%$ der Enzymaktivität des unbehandelten Ansatzes nachweisbar (s. Tab. 3). Bemerkenswerterweise ergaben analog durchgeführte Versuche mit Phosphoglucose-Isomerase aus Hefe (Boehringer Mannheim $\mathrm{GmbH}$ ), daß dieses Enzym selbst bei pH 3 über 5 Minuten voll aktiv bleibt. Nach der gleichen Zeit in $0,01 \mathrm{~N} \mathrm{HCl}$ waren noch $50 \%$ der $\mathrm{Ak}$ tivität beim Hefeenzym erhalten.

Für die Praxis ist also zur Ausschaltung der FructoseInterferenz Hämolyse der Blutprobe in 0,1 N Essigsäure die Methode der Wahl. In hoher Konzentration zugesetzte Fructose beeinflußt bei derart vorbehandelten Proben das Ergebnis der Glucosebestimmung nur noch unbedeutend (s. Abb. 3 und Tab. 4).

Tab. 4

Glucosebestimmung in Essigsäure-hämolysierten Blutproben mit und ohne Fructosezusatz $(5 \mathrm{~g} / \mathrm{l})$

\begin{tabular}{ccccc}
\hline & Proben-Nr. & \multicolumn{2}{c}{$\mathrm{g} / 1$} & $\mathbf{g} / 1$ \\
& - Fructose & + Fructose & \\
\hline & 1 & 0,82 & 0,82 & \\
& 2 & 1,07 & 1,09 & \\
3 & 0,54 & 0,57 & \\
\hline & 4 & 0,86 & 0,85 & \\
\hline
\end{tabular}

Anwendung der fluorimetrischen

Glucosebestimmung

\section{Eicbleurve}

Der Zusammenhang zwischen Fluoreszenzintensität und Glucosekonzentration ist im Meßbereich von 0-4 g/l linear (s. Abb. 4). Nach Abzug des Reagenzienleerwertes besteht daher direkte Proportionalität $z$ wischen Fluoreszenz und Glucosekonzentration.

\section{Präzision}

20 Bestimmungen der Glucosekonzentration eines Kontrollserums ergaben: $\overline{\mathrm{x}}=1,92 \mathrm{~g} / \mathrm{l} ; \mathrm{s}=0,03 \mathrm{~g} / \mathrm{l}$; $\mathrm{VK}=1,6 \%$.

\section{Ricbtigkeit}

Von 20 verschiedenen Blutproben wurde die Glucosekonzentration einerseits konventionell mit der Hexokinasemethode (8) photometrisch aus enteiweißtèm 


\section{Laborautomatisation - konsequent und zukunftssicher}

\section{Direkte Probenidentifikation On-line-Meßwerterfassung Real-time-Datenverarbeitung}
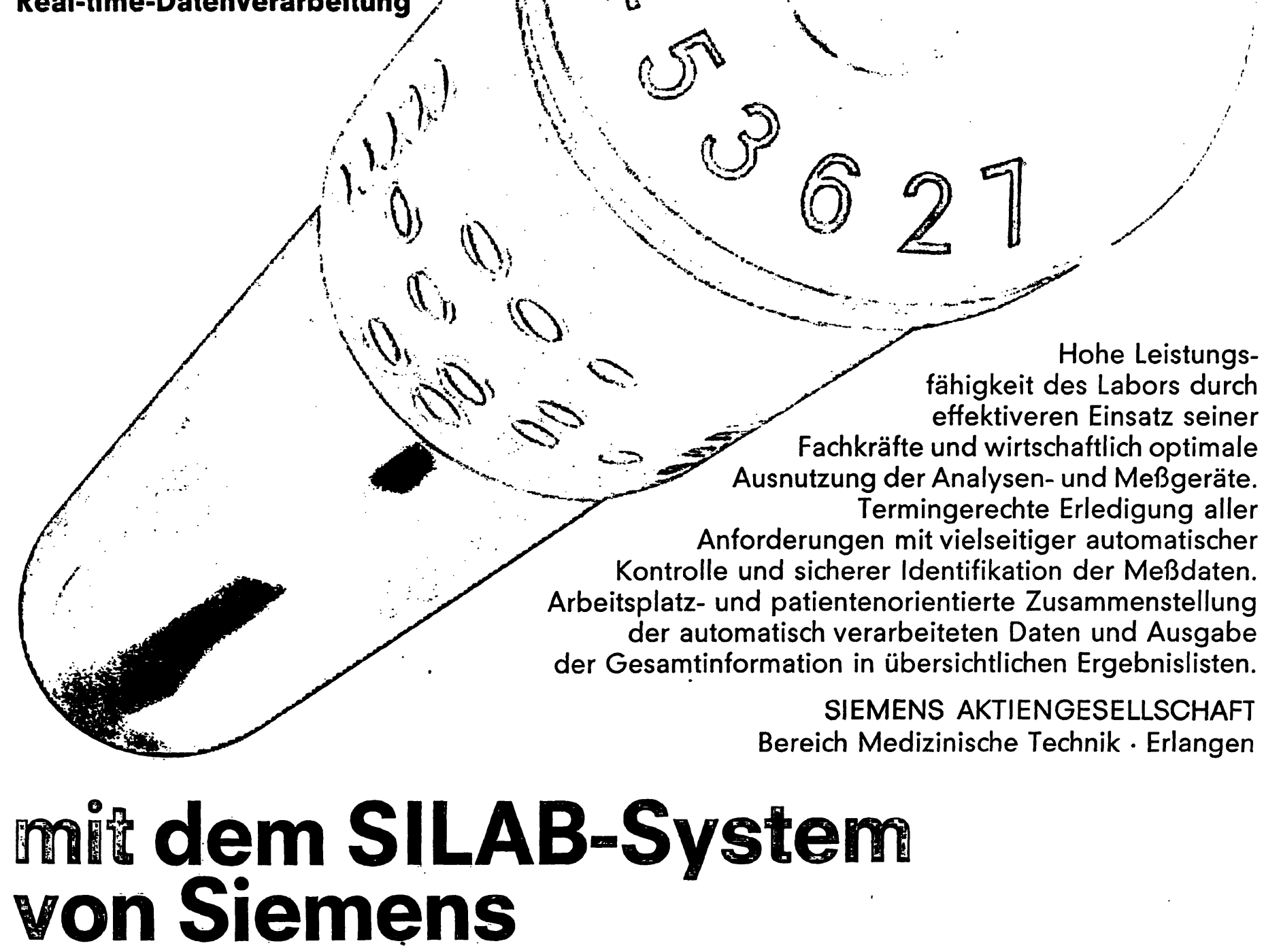


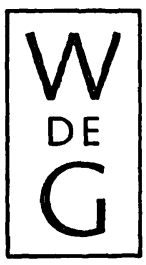

\section{Walter de Gruyter Berlin·New York}

\section{Proceedings \\ of the International Research Conference on Proteinase Inhibitors}

Munich, November 4-6, 1970

Edited by $\mathrm{H}$. Fritz and $\mathrm{H}$. Tschesche

XII, 304 pages. With 246 figures and

95 tables. 1971. Bound DM 108,-;

$\$ 31.75$
Contents:

Proteinase Inhibitors in Human Medicine Specific Isolation Methods - Inhibition Mechanism: Theories and Methods • Inhibitors of Plant Tissues - Basic Bovine Inhibitor and Related Inhibitors • Pancreatic Secretory Inhibitors - Seminal Inhibitors, Inhibitors and Fertilization. Inhibitors from Dog Submandibular Glands, Ascaris Lumbricoides and Leeches. Contributions by:

Birk, Coan, Cechová, Dlouhá-Keil, Feeney, Feinstein, Fink, Fritz, Greene, Haendle, Heimburger, Hochstraßer, Huber, Ikenaka, Kassell, Laskowski Jr., Laskowski Sr., Liener, Peanasky, Rigbi, Ryan, Schumacher, Shotton, Stevens, Tschesche, Werle, Zaneveld.

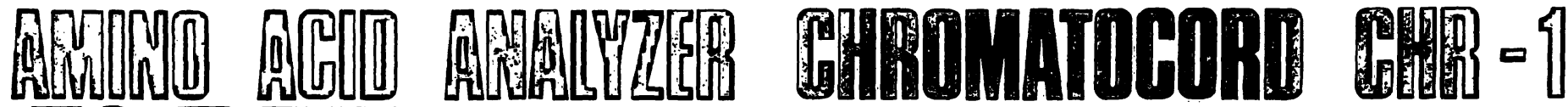

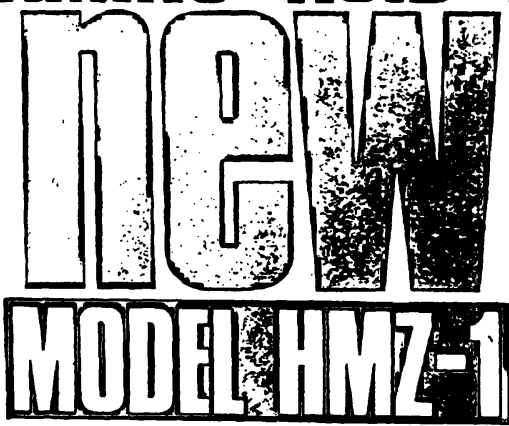

Semi Mikro Attachment Automatic Analyses on Picomol - Samples

Sensitivity: 20 Picomol
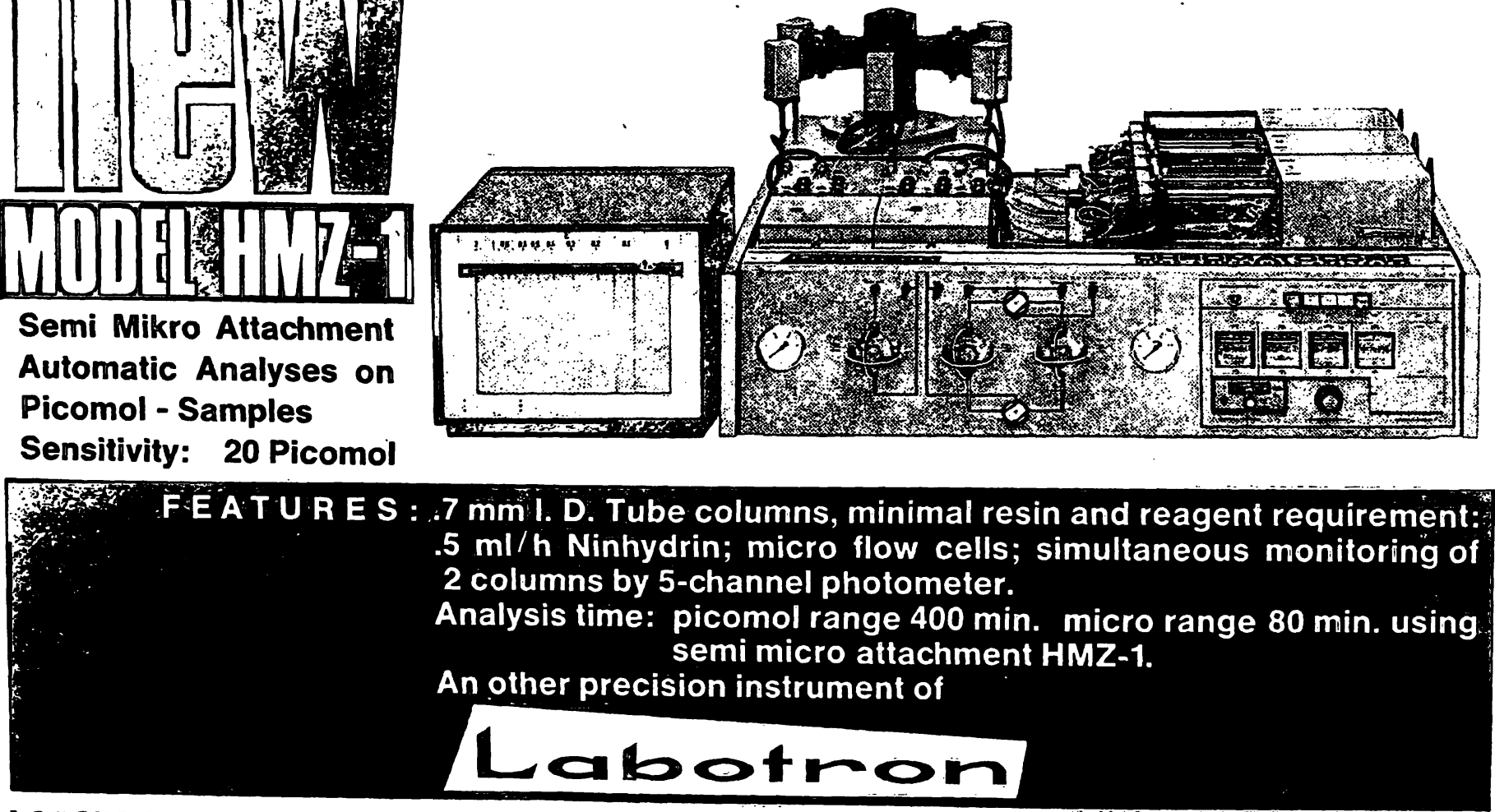

LABOTRON MESSTECHNIK GMBH 8191 GETING WOLFATSHAUSER STR.2 GEPMANY 


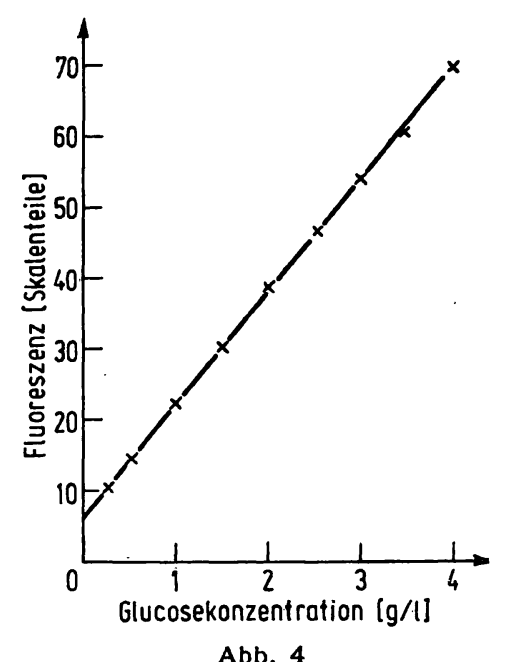

Abhängigkeit der Fluoreszenz von der Glucosekonzentration

Überstand, andererseits fluorimetrisch im Hämolysat bestimmt. Die sehr enge Korrelation zwischen den erhaltenen Ergebnissen zeigt Abbildung $5(r=0,999)$. Der $\operatorname{tg} \alpha$ für die errechnete Regressionsgerade liegt etwas unter dem theoretischen Wert von 1. Dazu ist $\mathrm{zu}$ bemerken, daß unsere photometrischen Bestimmungen auf Extinktionsmessungen im EppendorfSpektrallinienphotometer bei $366 \mathrm{~nm}$ beruhen. Es ist anzunehmen, daß die mit $\varepsilon_{\mathrm{NADPH}}=3,30 \cdot 10^{6} \mathrm{~cm}^{2} / \mathrm{Mol}$ errechneten Glucosewerte zu hoch liegen. Wir erhielten nämlich mit wäßr. Glucoselösungen im „optischen

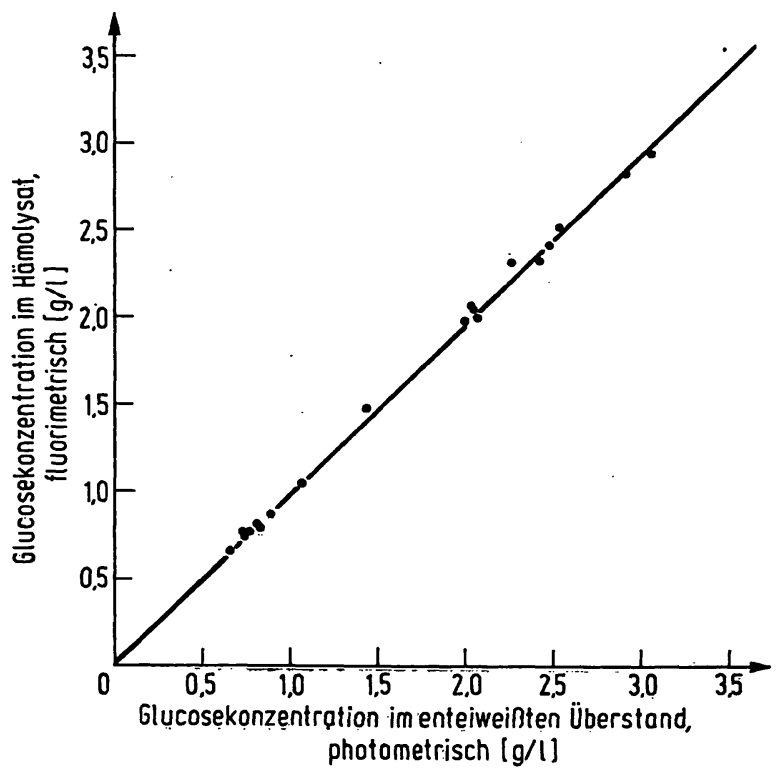

Abb. 5

Vergleich der Glucosewerte, die mit der klassischen Hexokinasemethode mit Enteiweißung (Abszisse) und der fluorimetrischen Methode im Hämolysat (Ordinate) bestimmt wurden $r=0,999 ; a=0,0019 ; b=0,970$

Test“" bei $366 \mathrm{~nm}$ Analysenergebnisse, die mehr als 100\% der Theorie entsprachen. Photometriert man denselben Bestimmungsansatz bei 334 und $366 \mathrm{~nm}$ und multi- pliziert mit den entsprechenden Berechnungsfaktoren, so ergeben sich bei 366 um $2,5-3,0 \%$ höhere Glucosewerte als bei $334 \mathrm{~nm}$. Ähnliche Beobachtungen wurden vor kurzem von anderer Seite mitgeteilt (9). Hierdurch dürfte die geringe systematische Abweichung der Ergebnisse beim Vergleich beider Methoden hinreichend erklärt sein.

\section{Wiederfindung}

Bei 5 verschiedenen Blutproben, denen $1,00 \mathrm{~g} / \mathrm{l}$ Glucose zugesetzt wurde, exgab sich eine durchschnittliche Wiederfindung von $101 \%$.

\section{Diskussion}

Das von BERNT und LACHENICHT vorgeschlagene sehr elegante Verfahren zur kontinuierlichen fluorimetrischen Glucosebestimmung im Hämolysat (5) gab uns die Anregung, die Besonderheiten der Hexokinasemethode bei Anwendung auf dieses Material eingehender zu studieren.

Nachdem es als Resultat dieser Untersuchungen gelungen ist, durch Inaktivierung der störenden Erythrocytenenzyme die Spezifität der Hexokinasemethode auch bei Glucosebestimmungen in hämolysierten Blutproben $z u$ erhalten, scheint uns der breiteren Anwendung der ausgearbeiteten Methode nichts im Wege zu stehen. Wir selbst verwerten die gewonnenen Erfahrungen für eine bei uns im Routinelaboratorium eingeführte Glucosebestimmung im Vollblut mit dem AutoAnalyzer (10).

Die in dieser Arbeit beschriebene manuelle Methode zur Glucosebestimmung im Hämolysat lehnt sich hinsichtlich der Bedingungen des Inkubationsansatzes an die von Scherstén und Tibbling (11) für Harnglucosebestimmungen empfohlene Vorschrift an. Der Vorteil des geringen Inkubationsvolumens liegt in der sehr ökonomischen Nutzung der Hexokinaseund Glucose-6-phosphat-Dehydrogenase-Aktivität. Aus Gründen der unterschiedlichen Enzymsättigung ist die Umsetzung der gleichen Glucosemenge im „konzentrierten Inkubationsansatz" (Volumen $=125 \mu \mathrm{l}$ ) viel rascher abgeschlossen, als wenn primär schon das für die Fluorimetrie günstige Endvolumen von etwa $10 \mathrm{ml}$ gewählt würde. Bei geringem Enzymverbrauch ist daher unsere fluorimetrische Glucosebestimmung nicht nur wegen des Fortfalls der Enteiweißung, sondern auch wegen der kürzeren Inkubationsdauer schneller durchführbar als die klassische photometrische Hexokinasemethode.

Frau Monika Kreine-Tebre hat durch ausgezeichnete technische Assistenz bei der Durchführung der Versuche wertvolle Hilfe geleistet.

\section{Literatur}

1. HAEckel, R., diese Z. 8, 480 (1970). - 2. Stork, H. und F. H. SchMidr, Klin. Wschr. 46, 789 (1968). - 3. BerGMEYER, H. U., E. Bernt, F. H. Schmidt und H. Stork, in: Methoden der enzymatischen Analyse, S. 1167. Hrsg. H. U. Bergmerbr, 2. Auf., Weinheim (1970). - 4. Rubin, M. und L. KNOtr, Clin. Chim. Acta, Amsterdam 18, 409 (1967). - 5. Bernt, E. 
und R. LACHENICHT, in: Methoden der enzymatischen Analyse, S. 1168. Hrsg. H. U. BergmeYer, 2. Auf., Weinheim (1970). 6. WeBB, J. L., Enzyme and Metabolic Inhibitors Vol. 3, S. $337 \mathrm{ff}$., New York and London (1966). - 7. Kahana, S. E., O. H. Lowry, D. W. Schulz, J. V. Passoneau und E. J. Crawford, J. biol. Chemistry 235, 2178 (1960). - 8. Scermid, F. H., Klin. Wschr. 39, 1244 (1961). - 9. HaEcked, R. und H. HAEckEL, Die Zuverlässigkeit der automatisierten Aktivitätsbestimmung der
Kreatinphosphokinase. Arbeitstagung der Dt. Ges. für Klin. Chemie, der Schweiz. Vereinig. für Klin. Chemie und der Osterr. Ges. für Klin. Chemie, Wien 1971. - 10. Manuskript in Vorbereitung. - 11. Schersstén, B. und G. Trbaning, Clin. Chim. Acta, Amsterdam 18, 383 (1967). - 12. Methoden der enzymatischen Analyse S. 462. Hrsg. H. U. BergmeYer, 2. Aufi, Weinheim (1970).

Dr. F. da Fonseca-Wollheim Institut für Klinische Chemie und Klinische Biochemie Klinikum Steglitz der FU Berlin 1 Berlin 45

Hindenburgdamm 30 